\title{
General Practitioner-Performed Compression Ultraso- nography for Diagnosis of Deep Vein Thrombosis of the Leg: A Multicenter, Prospective Cohort Study
}

\author{
Nicola Mumoli, MD \\ Josè Vitale, MD \\ Matteo Giorgi-Pierfranceschi, MD \\ Silvia Sabatini, MD \\ Renato Tulino, MD \\ Marco Cei, MD \\ Eugenio Bucherini, MD \\ Carlo Bova, MD \\ Daniela Mastroiacovo, MD \\ Alberto Camaiti, MD \\ Gerardo Palmiero, MD \\ Luca Puccetti, MD \\ Francesco Dentali, MD \\ for the PRACTICUS Study \\ Investigators

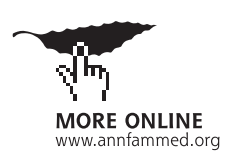

Conflicts of interest: authors report none.

\section{CORRESPONDING AUTHOR}

Nicola Mumoli, MD

Department of Internal Medicine

Ospedale Civile di Livorno

viale Alfieri 36

57100 Livorno, Italy

nimumoli@tiscali.it

\begin{abstract}
BACKGROUND Patients with suspected deep vein thrombosis (DVT) of the lower limb represent a diagnostic dilemma for general practitioners. Compression ultrasonography (US) is universally recognized as the best test of choice. We assessed the diagnostic accuracy of compression US performed by general practitioners given short training in the management of symptomatic proximal DVT.
\end{abstract}

METHODS From May 2014 to May 2016, we evaluated in a multicenter, prospective cohort study all consecutive outpatients with suspected DVT; bilateral proximal lower limb compression US was performed by general practitioners and by physicians expert in vascular US, each group blinded to the other's findings. In all examinations with a negative or nondiagnostic result, compression US was repeated by the same operator after 5 to 7 days. Inter-observer agreement and accuracy were calculated.

RESULTS We enrolled a total of 1,107 patients. The expert physicians diagnosed DVT in 200 patients, corresponding to an overall prevalence of $18.1 \%(95 \% \mathrm{Cl}$, 15.8\%-20.3\%). The agreement between the trained general practitioners and the experts was excellent (Cohen $\kappa=0.86 ; 95 \% \mathrm{Cl}, 0.84-0.88$ ). Compression US performed by general practitioners had a sensitivity of $90.0 \%(95 \% \mathrm{Cl}, 88.2 \%$ $91.8 \%)$ and a specificity of $97.1 \%(95 \% \mathrm{Cl}, 96.2 \%-98.1 \%)$ with a diagnostic accuracy for DVT of $95.8 \%$ (95\% Cl, 94.7\%-97.0\%).

CONCLUSIONS Our results suggest that, even in hands of physicians not expert in vascular US, compression US can be a reliable tool in the diagnosis of DVT. We found that the sensitivity achieved by general practitioners appeared suboptimal, however, so future studies should evaluate the implementation of proper training strategies to maximize skill.

Ann Fam Med 2017;15:535-539. https://doi.org/10.1370/afm.2109.

\section{INTRODUCTION}

$\mathrm{P}$ atients with clinical signs and symptoms of deep vein thrombosis (DVT) of the lower limbs represent a diagnostic dilemma for general practitioners. ${ }^{1}$ Reliance on clinical findings alone can lead to misdiagnosis, unnecessary exposure to anticoagulant therapy, and high associated costs. Rapid and accurate diagnosis of DVT is therefore needed to start prompt anticoagulation therapy and reduce the risk of potentially fatal pulmonary embolism. ${ }^{2,3}$ For these reasons, in general practice, the optimal diagnostic strategy for DVT has long been debated: the Wells score is not accurate enough for use in primary care, ${ }^{4}$ but strategies that require D-dimer testing ${ }^{5}$ are not always accessible. Compression ultrasonography (US) is considered the more widespread method of choice for confirming or ruling out the diagnosis of DVT, and its use has been validated in several prospective studies ${ }^{3,6-8}$; the potential advantage of this technique is its universal availability in different settings, by different operators, and with almost all types of US scanners. Some recent studies have demonstrated a similar accuracy of compression US performed 
by hospitalists, radiologists, emergency physicians, or trained nurses. ${ }^{9-11}$ A more rapid diagnosis, directly obtained by general practitioners in primary care, could likely improve appropriate management of DVT, avoiding in-hospital evaluations and any pretest scores or laboratory evaluations. We undertook a prospective, multicenter cohort study to assess the diagnostic accuracy of compression US performed by general practitioners given a short course of training in the diagnosis of suspected DVT of the lower limbs.

\section{METHODS}

Our study, PRACTICUS (general PRACTItionerperformed Compression UltraSonography in the diagnosis of proximal symptomatic deep vein thrombosis of the lower limbs), was conducted in 18 primary care ambulatory clinics and 5 vascular reference clinics in Italy. We report its findings according to the Standards for Reporting of Diagnostic Accuracy Studies (STARD) initiative, which established reporting guidelines for diagnostic accuracy studies ${ }^{12}$ (for details, see Supplemental Appendix, available at http://www. AnnFamMed.org/content/15/6/535/supp1/DC1/).

The study protocol was approved by the local institutional review board and registered at ClinicalTrials. gov as NCT02114983.

\section{Study Eligibility}

Between May 2014 and May 2016, general practitioners, in their own primary care offices, prospectively evaluated all consecutive ambulatory patients who sought care with a clinically suspected DVT of the lower limbs. The probability of DVT was not calculated with the Wells score, but was assessed by each physician, according to his or her discretion, on the basis of the patient's medical history and clinical picture. When the differential diagnosis determined that DVT was one of the most probable diagnoses, the patient became eligible for the study. Written informed consent was obtained from all study participants.

Exclusion criteria were a previous objectively documented episode of venous thromboembolism, predominant symptoms of pulmonary embolism, age younger than 18 years, pregnancy, life expectancy of less than 3 months, ongoing mandatory anticoagulant therapy, symptoms lasting for more than 2 weeks, geographic inaccessibility for follow-up, anticipated poor compliance, and declining to provide written informed consent.

\section{Study Design}

All eligible patients underwent 2 compression US examinations: the first performed in general ambulatory care by a trained general practitioner and the other, within
2 hours, performed in a local vascular center clinic by a physician expert in vascular US. The 2 examinations were performed separately and in a blinded manner with respect to each other. US examinations with negative or unclear results were repeated by the same operator after 5 to 7 days independently of the other group.

At the second examination, physician experts made a definitive diagnosis regarding DVT: present or absent. Patients were instructed not to communicate results of their compression US or initiation of anticoagulant therapy to the other investigators to maintain blinding. All US examination findings were maintained by individual physicians in their own databases and transmitted to an external researcher (J.V.) at the end of recruitment.

\section{Study Physicians}

A complete list of the investigators participating in the PRACTICUS study is provided in the Supplemental Appendix (available at http://www.AnnFamMed.org/ content/15/6/535/suppl/DC1/).

Before the study began, all general practitioners received 2 months of formal training in compression US by local senior physicians expert in vascular US. This course consisted of 12 hours (6 hours per month) of didactic lecture followed by 30 hours ( 15 hours per month) of practice on patients in vascular center clinics and 8 hours ( 4 hours per month) of vascular US image review (Supplemental Appendix, available at http:// www.AnnFamMed.org/content/15/6/535/suppl/DC1/).

All participating physician experts in vascular US were board certified and had at least 10 years of experience in vascular US, performing a minimum of 500 examinations per year (Supplemental Appendix, available at http://www.AnnFamMed.org/content/15/6/535/suppl/ DC1/). Their final interpretation of compression US results was considered the reference test for our study.

\section{Compression US Procedure}

Enrolled patients were examined with the US machine routinely used by the operator (Supplemental Appendix, http://www.AnnFamMed.org/content/15/6/535/suppl/ $\mathrm{DC} 1 /$ ) with a 5 - to $7.5-\mathrm{MHz}$ linear probe. The examination was performed with 2-point compression and interpreted according to described methods. ${ }^{13,14}$ The proximal venous system of the common and superficial femoral vein was examined first, with the patient lying supine. The popliteal vein to its trifurcation was evaluated with the patient in the prone position or in a (left or right) lateral decubitus position, eventually using augmentation maneuvers to enhance vessel visualization.

The protocol for the compression US consisted of bilateral sequential compressions along the femoral veins and along the popliteal veins. All compressions were performed using B-mode imaging with transverse scans. 
The compression US technique was performed from the groin area to the popliteal fossa by moving the transducer distally and applying gentle compression along the deep venous system. A copy of the compression US imaging was recorded for all vascular sites. The diagnostic criterion for DVT was inability to fully compress the lumen of the vein in the transverse plane. ${ }^{13,14}$

\section{Sample Size Calculation and Statistical Analysis}

On the basis of evidence in the literature ${ }^{3}$ showing a prevalence of proximal DVT in outpatients of about $17 \%$ to $19 \%$, we calculated a sample size of at least 1,000 patients to obtain reliable results with a low margin of uncertainty. Continuous variables were summarized as means and SDs, and noncontinuous variables as frequencies and percentages. The Student $t$ test and $\chi^{2}$ test were used for comparisons. A 2-tailed $P$ value less than .05 indicated statistical significance. Inter-observer agreement was analyzed using the weighted Cohen $\kappa$ coefficient and reported as a point estimate with a $95 \% \mathrm{CI} .{ }^{15}$ The $\kappa$ coefficient values were interpreted as follows: $<.20$ indicates poor agreement; .21 to .40 fair agreement; .41 to .60 moderate agreement; .61 to .80 good agreement; and 0.81 to 1 excellent agreement. ${ }^{15}$ We calculated diagnostic accuracy, sensitivity, specificity, positive and negative predictive values, and likelihood ratios of general practitioner-performed compression US with their 95\% CIs, using the expert vascular physician diagnosis as the reference test. Finally, the posttest probability of having or not having DVT was calculated. Statistical analyses were performed using SPSS 15.0 software (SPSS Inc).

\section{RESULTS}

\section{Patient and DVT Characteristics}

From May 2014 to May 2016, a total of 1,247 consecutive outpatients with clinically suspected DVT were referred, of whom 109 (9\%) were excluded because they met 1 or more predefined exclusion criteria (Figure 1). Another 31 (3\%) of the patients were excluded from the analysis for violation of the blinding protocol, having communicated the outcome of their compression US or their initiation of anticoagulant therapy.

The characteristics of the remaining 1,107 patients, who were evaluated by 18 general practitioners and 8 expert vascular US physicians, are summarized in Table 1. Comparing the patients with and without DVT, there was no significant difference in mean age or sex $(P=.45$ and .57 , respectively).

Physician experts diagnosed DVT in 200 patients, for an overall prevalence of $18.1 \%$ (95\% CI, $15.8 \%$ $20.3 \%$ ). Femoral veins were occluded in 104 patients, while popliteal veins were occluded in 152 patients; in 56 patients $(28 \%)$, both were occluded.

\section{Outcomes}

Results of compression US performed by general practitioners are summarized in Table 2. Overall, agreement between the 2 groups of physicians was excellent, with a weighted Cohen $\kappa$ statistic of $0.86(95 \% \mathrm{CI}$, 0.84-0.88). Of note, agreement on the diagnosis of DVT was almost perfect when both femoral and popliteal veins were affected ( $\kappa=0.99 ; 95 \% \mathrm{CI}, 0.98-0.99)$, whereas it was lower when only the popliteal vein was involved ( $\kappa=0.88 ; 95 \% \mathrm{CI}, 0.86-0.90)$.

Using the final diagnosis recorded by the physicians expert in vascular US as the reference test, the diagnostic accuracy of general practitioners was $95.8 \%$ (95\% CI, 94.7\%-97.0\%), with a sensitivity of $90.0 \%$ (95\% CI, $88.2 \%-91.8 \%)$, a specificity of $97.1 \%(95 \%$ CI, 96.2\%-98.1\%), a positive predictive value of $87.4 \%$

\section{Figure 1. Patient screening and study inclusion.}

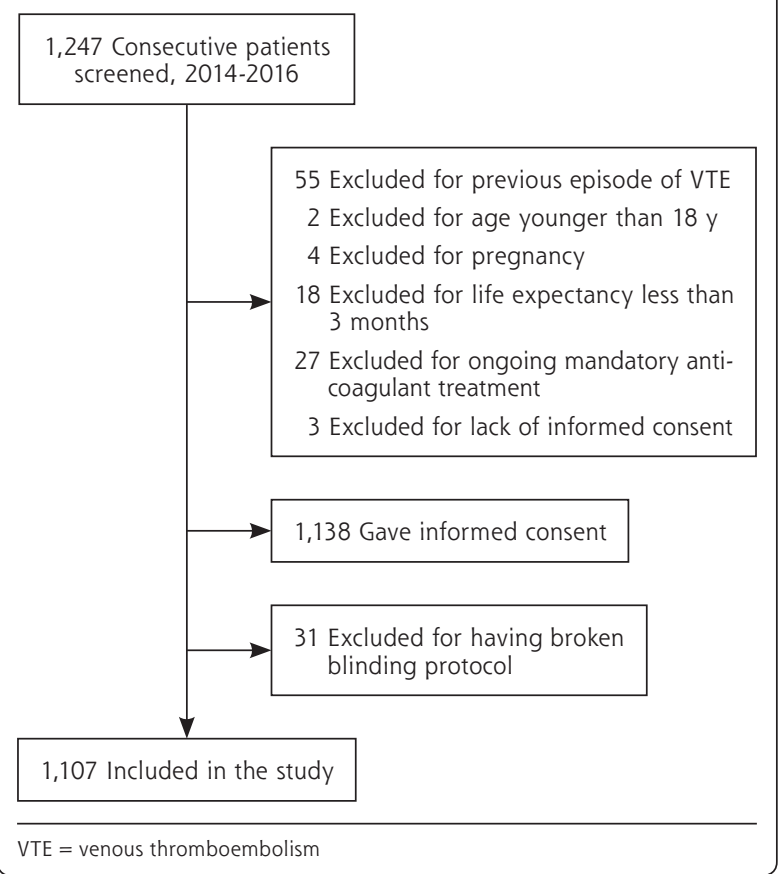

Table 1. Patient Demographic and Clinical Characteristics $(\mathrm{N}=1,107)$

\begin{tabular}{lcc}
\hline Characteristics & $\begin{array}{c}\text { DVT } \\
\text { (n= 200) }\end{array}$ & $\begin{array}{c}\text { No DVT } \\
(\mathbf{n}=\mathbf{9 0 7})\end{array}$ \\
\hline Age, mean (SD), [range], y & $63.6( \pm 15.2)$ & $63.8( \pm 14.9)$ \\
& {$[20-101]$} & {$[19-98]$} \\
Men, No. (\%) & $104(52)$ & $587(53)$ \\
Site of DVT & $104(52)$ & $n / a$ \\
Femoral vein, No. (\%) & $152(76)$ & $n / a$ \\
Popliteal vein, No. (\%) & $56(28)$ & $n / a$ \\
Both, No. (\%) & \\
\hline DVT = deep vein thrombosis; n/a = not applicable. & \\
\hline
\end{tabular}


Table 2. Diagnostic Performance of General Practitioner-Performed Compression US for Proximal DVT

\begin{tabular}{|c|c|c|c|c|c|c|}
\hline DVT Site & $\begin{array}{l}\text { Sensitivity, \% } \\
(95 \% \mathrm{Cl})\end{array}$ & $\begin{array}{c}\text { Specificity, \% } \\
(95 \% \text { Cl) }\end{array}$ & $\begin{array}{l}\text { PPV, \% } \\
(95 \% \text { Cl) }\end{array}$ & $\begin{array}{l}\text { NPV, \% } \\
(95 \% \text { Cl) }\end{array}$ & $\begin{array}{c}\text { Accuracy, } \% \\
(95 \% \mathrm{Cl})\end{array}$ & $\begin{array}{l}\text { Cohen } \kappa_{,} \% \\
(95 \% \mathrm{Cl})\end{array}$ \\
\hline Overall & $90.0(88.2-91.8)$ & $97.1(96.2-98.1)$ & $87.4(85.4-89.3)$ & $97.8(96.9-98.6)$ & $95.8(94.7-97.0)$ & $0.86(0.84-0.88)$ \\
\hline Femoral & $95.2(93.9-96.5)$ & $98.8(98.2-99.4)$ & $89.2(87.4-91.0)$ & 99.5 (99.1-99.9) & 98.5 (97.7-99.2) & $0.91(0.89-0.93)$ \\
\hline Popliteal & $89.5(87.7-91-3)$ & $98.4(97.7-99.2)$ & 90.1 (88.3-91.8) & $98.3(97.6-99.1)$ & $97.2(96.2-98.2)$ & $0.88(0.86-0.90)$ \\
\hline Both & $98.7(98.0-99.3)$ & 100 (99.9-100) & $100(99.9-100)$ & $99.9(99.7-100)$ & 99.9 (99.7-100) & $0.99(0.98-0.99)$ \\
\hline
\end{tabular}

DVT = deep vein thrombosis; NPV = negative predictive value; PPV = positive predictive value; US = ultrasound.

Note: Diagnosis of physician expert in vascular US was the reference test.

(95\% CI, 85.4\%-89.3\%), and a negative predictive value of $97.8 \%$ (95\% CI, 96.9\%-98.6\%) (Table 2).

Positive and a negative likelihood ratios indicated that with a prevalence of DVT in our study of $18.1 \%$, the posttest probability of having this disorder when general practitioner-performed compression US was positive was $87 \%$, and the posttest probability of not having the disorder when general practitioner-performed compression US was negative was about $2 \%$.

\section{DISCUSSION}

Every year, about 2 million patients in the United States undergo US examination in the hospital for suspected DVT. ${ }^{16,17}$ This estimate is likely to increase because of aging of the population and more widespread awareness of the health risks of venous thromboembolism. ${ }^{17}$ Data from emergency departments in the United States show that the number of investigations appears to be growing much more than the number of operators dedicated to performing them. ${ }^{16,17}$ Although US evaluation for DVT can be successfully performed in just a few minutes, at the patient's bedside, and by nonvascular specialists, ${ }^{11}$ the availability of physicians trained in compression US is still inadequate. In the future, with the diffusion of point-of-care US, an increasing number of physicians will have the technology available to perform clinical ultrasonography. ${ }^{18}$

Our findings suggests that in the diagnosis of DVT, general practitioners might be a potential alternative to vascular experts because they achieve an excellent agreement and an overall high accuracy of $95.8 \%$. Unfortunately, their sensitivity still appears suboptimal, but it may improve with practice and experience. In other fields of diagnostic US, this expertise has already proven to be successful..$^{19-21}$ The general practitioners in our study had minimal training, which probably influenced their performance, at least in the initial period. Future studies should therefore focus not on the type of operator (ie, physician, nurse, technician), but on the training and technical expertise, evaluating the change in accuracy over time and with increasing number of examina- tions. If these studies confirm our preliminary results, a training program in compression US should be offered to general practitioners, because it could improve the quality of health services and promote more rapid and appropriate management in patients with suspected DVT. We speculate that such training would also reduce waiting lists in hospitals and probably costs as well.

On a more general note, our study corroborates the universal utility of compression US performed by any operator without in-hospital evaluation, D-dimer testing, and use of any pretest scores. We imagine the utility could extend to varied scenarios where there are no expert physicians specialized in US or radiology; to geographic areas where resources are limited, where vascular laboratories are not available on a 24 -hour basis, and where patients can be lost to follow-up; or to overcrowded emergency and medicine departments.

Our study has both strengths and limitations. We limited the examination to proximal veins and did not perform the whole-leg compression US. Although proximal lower limb compression US has to be repeated in cases of negative results in patients at high risk for DVT, whole-leg compression US is more time consuming and more difficult to learn, and these 2 procedures appear to be equally effective. ${ }^{2,4,5}$ In addition, we did not compare general practitioner-performed compression US with venography because the latter is unsuitable for routine use in patients with suspected $\mathrm{DVT}^{2}$ and has been progressively abandoned. Indeed, in the last decade, US has largely replaced contrast phlebography as the definitive diagnostic test for DVT, ${ }^{2-6}$ and many studies have demonstrated that duplex ultrasonography, with the criterion of vein compressibility and with spectral/color Doppler support, is a highly accurate, simple, objective, and reproducible noninvasive diagnostic method in outpatients with clinically suspected DVT. ${ }^{2-6}$

We used several strategies to minimize the risk of bias: patients were enrolled consecutively and prospectively; the protocol for blinding was rigidly respected; the expert physicians were highly experienced in vascular US, and we used a multicenter design, obtaining an adequate sample size and involving a substantial number 
of general practitioners. Furthermore, to increase the external validity of our findings and to better reflect the real-world management of these patients, we included only individuals with a suspected first episode of DVT, assessed simply with a clinical judgment and without the aid of scores or laboratory tests. On the other hand, we are aware that observational studies have a high risk of bias that may affect the internal validity of their results. Furthermore, although all the general practitioners were trained, they performed their examination mostly outside of the hospital using different US machines, and their practice was limited to the study protocol.

In conclusion, our results suggest for the first time that compression US performed by trained general practitioners may be an accurate alternative for diagnosis of suspected proximal DVT. Although this approach may help reduce the time to diagnosis and then optimize proper management, future studies should include more rigorous assessment to maximize its accuracy.

To read or post commentaries in response to this article, see it online at http://www.AnnFamMed.org/content/15/6/535.

Key words: general practitioner; primary care; deep vein thrombosis; compression ultrasound; accuracy; diagnosis; practice-based research

Submitted December 9, 2016; submitted, revised, March 30, 2017; accepted April 24, 2017.

Author Affiliations: Department of Internal Medicine, Ospedale Civile di Livorno, Livorno, Italy (Mumoli, Cei, Camaiti, Palmiero); Department of Internal Medicine, Ospedale Beata Vergine di Mendrisio, Ticino, Switzerland (Vitale); Emergency Department, Ospedale della Val d'Arda, Piacenza, Italy (Giorgi-Pierfranceschi); Primary Ambulatory Care, Livorno, Italy (Sabatini); Primary Care, Porto Santo Stefano, Grosseto, Italy (Tulino); Department of Vascular Medicine, ASL Di Romagna, Ravenna, Italy (Bucherini); Department of Internal Medicine, Azienda Ospedaliera, Cosenza, Italy (Bova); Angiology Unit, ASL 1 Avezzano Sulmona L'Aquila, Avezzano (AQ), Italy (Mastroiacovo); Primary Ambulatory Care, Pisa, Italy (Puccetti); Department of Internal Medicine, Ospedale di Circolo, Varese, Varese, Italy (Dentali).

Author contributions: N.M., J.V., S.S., and R.T. were responsible for study concept and design; N.M., M.G-P., E.B., S.S., R.T., D.M., L.P., C.B., A.C., G.P., and M.C. for data acquisition; N.M. and J.V. for database handling and updating; N.M., J.V., and F.D. for statistical analysis; N.M., J.V., M.G-P., and M.C. for drafting of the manuscript; N.M., J.V., and F.D. for interpretation of results; and F.D. for critical revision of the manuscript for important intellectual content. All authors had full access to all of the data in the study and take responsibility for the integrity of the data and the accuracy of the data analysis.

Supplemental materials: Available at http://www.AnnFamMed. org/content/15/6/535/suppl/DC1/.

\section{References}

1. Qaseem A, Snow V, Barry P, et al; Joint American Academy of Family Physicians/American College of Physicians Panel on Deep Venous Thrombosis/Pulmonary Embolism. Current diagnosis of venous thromboembolism in primary care: a clinical practice guideline from the American Academy of Family Physicians and the American College of Physicians. Ann Intern Med. 2007;146(6):454-458.
2. Kearon C. Natural history of venous thromboembolism. Circulation. 2003;107(23)(Suppl 1):122-130.

3. Bates SM, Jaeschke R, Stevens SM, et al. Diagnosis of DVT: Antithrombotic Therapy and Prevention of Thrombosis, 9th ed: American College of Chest Physicians Evidence-Based Clinical Practice Guidelines. Chest. 2012;141(2 Suppl):e351S-e418S.

4. Oudega R, Hoes AW, Moons KGM. The Wells rule does not adequately rule out deep venous thrombosis in primary care patients. Ann Intern Med. 2005;143(2):100-107.

5. Büller HR, Ten Cate-Hoek AJ, Hoes AW, et al.; AMUSE (Amsterdam Maastricht Utrecht Study on thromboEmbolism) Investigators. Safely ruling out deep venous thrombosis in primary care. Ann Intern Med. 2009;150(4):229-235.

6. Kearon C, Julian JA, Newman TE, Ginsberg JS. Noninvasive diagnosis of deep venous thrombosis. McMaster Diagnostic Imaging Practice Guidelines Initiative. Ann Intern Med. 1998;128(8):663-677.

7. Bernardi E, Camporese G, Büller HR, et al.; Erasmus Study Group. Serial 2-point ultrasonography plus D-dimer vs whole-leg colorcoded Doppler ultrasonography for diagnosing suspected symptomatic deep vein thrombosis: a randomized controlled trial. JAMA. 2008;300(14):1653-1659.

8. Goodacre S, Sampson F, Thomas S, van Beek E, Sutton A. Systematic review and meta-analysis of the diagnostic accuracy of ultrasonography for deep vein thrombosis. BMC Med Imaging. 2005;5:6.

9. Pearson SD, Polak JL, Cartwright S, McCabe-Hassan S, Lee TH, Goldhaber SZ. A critical pathway to evaluate suspected deep vein thrombosis. Arch Intern Med. 1995;155(16):1773-1778.

10. Pomero F, Dentali F, Borretta V, et al. Accuracy of emergency physician-performed ultrasonography in the diagnosis of deep-vein thrombosis: a systematic review and meta-analysis. Thromb Haemost. 2013;109(1):137-145.

11. Mumoli N, Vitale J, Cocciolo M, et al. Accuracy of nurse-performed compression ultrasonography in the diagnosis of proximal symptomatic deep vein thrombosis: a prospective cohort study. J Thromb Haemost. 2014;12(4):430-435.

12. Bossuyt PM, Reitsma JB, Bruns DE, et al.; STARD Group. Towards complete and accurate reporting of studies of diagnostic accuracy: the STARD initiative. Fam Pract. 2004;21(1):4-10.

13. Lensing AWA, Prandoni P, Brandjes $D$, et al. Detection of deep-vein thrombosis by real-time B-mode ultrasonography. $N$ Engl J Med. 1989;320(6):342-345.

14. Zierler BK. Ultrasonography and diagnosis of venous thromboembolism. Circulation. 2004;109(12)(Suppl 1):19-114.

15. Landis JR, Koch GG. The measurement of observer agreement for categorical data. Biometrics. 1977;33(1):159-174.

16. Barnes GD, Gafoor S, Wakefield T, Upchurch GR Jr, Henke P, Froehlich JB. National trends in venous disease. J Vasc Surg. 2010; 51(6):1467-1473.

17. Deitelzweig SB, Johnson BH, Lin J, Schulman KL. Prevalence of clinical venous thromboembolism in the USA: current trends and future projections. Am J Hematol. 2011;86(2):217-220.

18. Mengel-Jørgensen T, Jensen MB. Variation in the use of point-of-care ultrasound in general practice in various European countries. Results of a survey among experts. Eur J Gen Pract. 2016;22(4):274-277.

19. Yazbek J, Raju SK, Ben-Nagi J, Holland TK, Hillaby K, Jurkovic D. Effect of quality of gynaecological ultrasonography on management of patients with suspected ovarian cancer: a randomised controlled trial. Lancet Oncol. 2008;9(2):124-131.

20. Menon U. Gynaecological ultrasonography: expertise counts. Lancet Oncol. 2008;9(2):88-89.

21. Brulhart L, Ziswiler HR, Tamborrini G, Zufferey P; SONAR/SCQM programmes. The importance of sonographer experience and machine quality with regards to the role of musculoskeletal ultrasound in routine care of rheumatoid arthritis patients. Clin Exp Rheumatol. 2015;33(1):98-101. 